\title{
Influence of Casein kinase II inhibitor CX- 4945 on BCL6-mediated apoptotic signaling in B-ALL in vitro and in vivo
}

Anna Richter ${ }^{1}$, Sina Sender ${ }^{1}$, Annemarie Lenz ${ }^{1}$, Rico Schwarz², Burkhard Hinz ${ }^{2}$, Gudrun Knuebel ${ }^{1}$, Anett Sekora ${ }^{1}$, Hugo Murua Escobar ${ }^{1}$, Christian Junghanss ${ }^{1 *}$ (i) and Catrin Roolf ${ }^{1}$

\begin{abstract}
Background: Casein kinase II (CK2) is involved in multiple tumor-relevant signaling pathways affecting proliferation and apoptosis. CK2 is frequently upregulated in acute B-lymphoblastic leukemia (B-ALL) and can be targeted by the ATP-competitive CK2 inhibitor CX-4945. While reduced proliferation of tumor entities including B-ALL after CX-4945 incubation has been shown in vitro and in vivo, the detailed way of action is unknown. Here, we investigated the influence on the PI3K/AKT and apoptosis cascades in vivo and in vitro for further clarification.

Methods: A B-ALL xenograft model in NSG mice was used to perform in vivo longitudinal bioluminescence imaging during six day CX-4945 treatment. CX-4945 serum levels were determined at various time points. Flow cytometry of bone marrow and spleen cells was performed to analyze CX-4945-induced effects on tumor cell proliferation and distribution in B-ALL engrafted mice. ALL cells were enriched and characterized by targeted RNA sequencing. In vitro, B-ALL cell lines SEM, RS4;11 and NALM-6 were incubated with CX-4945 and gene expression of apoptosis regulators $B C L 6$ and $B A C H 2$ was determined.

Results: In B-ALL-engrafted mice, overall tumor cell proliferation and distribution was not significantly influenced by CK2 inhibition. CX-4945 was detectable in serum during therapy and serum levels declined rapidly after cessation of CX-4945. While overall proliferation was not affected, early bone marrow and spleen blast frequencies seemed reduced after CK2 inhibition. Gene expression analyses revealed reduced expression of anti-apoptotic oncogene $B C L 6$ in bone marrow blasts of CX-4945-treated animals. Further, BCL6 protein expression decreased in B-ALL cell lines exposed to CX-4945 in vitro. Surprisingly, levels of BCL6 opponent and tumor suppressor BACH2 also declined after prolonged incubation. Simultaneously, increased phosphorylation of direct CK2 target and tumor initiator AKT was detected at respective time points, even in initially PAKT-negative cell line NALM-6.

Conclusions: The CK2 inhibitor CX-4945 has limited clinical effects in an in vivo B-ALL xenograft model when applied as a single drug over a six day period. However, gene expression in B-ALL cells was altered and suggested effects on apoptosis via downregulation of BCL6. Unexpectedly, the BCL6 opponent BACH2 was also reduced. Interactions and regulation loops have to be further evaluated.
\end{abstract}

Keywords: B-ALL, Apoptosis, CK2, CX-4945, BCL6, BACH2, CDC42, AKT, Pharmakokinetic

\footnotetext{
* Correspondence: Christian.junghanss@med.uni-rostock.de:

onkologie@med.uni-rostock.de

${ }^{1}$ Department of Medicine, Clinic III - Hematology, Oncology, Palliative

Medicine, Rostock University Medical Center, Ernst-Heydemann-Strasse 6,

18057 Rostock, Germany

Full list of author information is available at the end of the article
}

(c) The Author(s). 2020 Open Access This article is distributed under the terms of the Creative Commons Attribution 4.0 International License (http://creativecommons.org/licenses/by/4.0/), which permits unrestricted use, distribution, and

reproduction in any medium, provided you give appropriate credit to the original author(s) and the source, provide a link to the Creative Commons license, and indicate if changes were made. The Creative Commons Public Domain Dedication waiver (http://creativecommons.org/publicdomain/zero/1.0/) applies to the data made available in this article, unless otherwise stated. 


\section{Background}

Casein kinase II (CK2) is a constitutively active, ubiquitously expressed serine/threonine kinase aberrantly activated in numerous solid and hematological tumors including acute B-lymphoblastic leukemia (B-ALL) [1]. CK2 phosphorylates a variety of target proteins with numerous functions involved in cell cycle regulation, cell growth, proliferation, transcription, translation and apoptosis. It thus influences pathways involved in tumorigenesis like PI3K/AKT, JAK/STAT and NFkB [2, 3]. CK2 acts via the inactivation of tumor suppressor genes PTEN and IKZF1 (Ikaros) as well as stimulating proliferation and cellular growth in lymphoid malignancies including B-ALL [4]. Further, the CK2-mediated induction of apoptotic pathways as an additional mode of action has been studied in solid tumors and acute myeloid leukemia (AML) [5-8] but remains largely uninvestigated in B-ALL so far.

CX-4945 is a selective, ATP competitive CK2 inhibitor and currently under investigation in clinical studies for renal tumors, cholangiocarcinoma, basal cell carcinoma and medulloblastoma. Our group as well as others have previously shown that CX-4945 inhibits tumor cell proliferation and metabolic activity in vitro and in vivo for numerous neoplastic entities including B-ALL $[1,9,10]$. Several mechanisms have been discussed to identify the inhibitor's anti-proliferative mode of action. These include intervention or modification of signaling pathways like PI3K/AKT, DNA repair response, angiogenesis, splicing regulation, stress-induced cell death or epigenetic modulation [10-16]. Nevertheless, it is still unknown how anti-leukemic effects are evoked in B-ALL. Induction of CK2-mediated apoptotic cascades or inhibition of anti-apoptotic pathways by CX-4945 might be mechanisms involved.

It has been demonstrated that incubation of B-ALL cells with CX-4945 induces apoptosis, possibly via increased cellular stress or inhibition of NFkB signaling [10, 11, 17]. So far it has not been investigated if in vitro effects are also present if CX-4945 is applied in vivo in B-ALL models. Further, little is known about which signaling molecules are involved in CX-4945-induced pro-apoptotic mechanisms and how those cascades are regulated during therapeutic approaches. We recently reported CX-4945induced effects in B-ALL xenografts [10]. In this follow-up study, we investigate the early molecular mechanisms of CK2 inhibitor CX-4945 on B-ALL. We aim to explore whether and how apoptotic processes play a role for the anti-leukemic properties of CX-4945.

\section{Methods}

Animal studies

NOD scid gamma mice (NOD.Cg-Prkdc $c^{\text {scid }} I l 2 r g^{t m 1 W j l} /$ SzJ, NSG, Charles River Laboratories, Sulzfeld, Germany) were bred and housed under specific pathogen-free conditions with access to water and standard chow ad libitum. All experiments were carried out in a laboratory setting and no intervention was performed within the animal housing and breeding rooms. Only healthy female animals aged 8 to 14 weeks and 19.1-27.7 g weight were included in the experiments. Study group sizes were four animals per time point and intervention group. Experiments were approved by the review board of the federal state Mecklenburg-Vorpommern, Germany (reference number: LALLF MV/7221.3-1.1002/15).

Study endpoints for all mice used are listed in Additional File 1: Table S1. Female mice were i.v. injected with $2.5 \times$ $10^{6}$ SEM cells stably transduced with GFP and enhanced firefly luciferase (ffLuc). Transfection was performed using the pCDH-EF1-MCS-T2A-copGFP vector (System Biosciences, Mountain View, CA, USA) using EcoRI and BamHI as previously described [18]. SEM-GFP-ffluc cells were kindly provided by Prof. Irmela Jeremias (Helmholtz Center Munich, Germany). Lentivirus production and cell transduction were carried out as described before [19]. Tumor cell engraftment was evaluated 7 days after injection via bioluminescence imaging (BLI) using the NightOWL LB 983 in vivo Imaging System (Berthold Technologies, Bad Wildbach, Germany) and Indigo software (Berthold Technologies, version 1.04). For detection, animals were intraperitoneally injected with $4.5 \mathrm{mg}$ D-Luciferin (GOLDbiotechnology, St. Louis, MO, USA), anesthetized with ketamine $(75 \mathrm{mg} / \mathrm{kg})$ and xylazine $(5 \mathrm{mg} / \mathrm{kg})$ and imaged in dorsal and ventral position $(60 \mathrm{~s}$ exposure, $560 \mathrm{~nm}$ emission). Mice were randomized based on weight, age and tumor cell engraftment on d7. CX-4945 was dissolved in $0.9 \%$ saline containing 5\% DMSO. Animals were simultaneously treated with either vehicle (isotonic saline supplemented with $5 \%$ DMSO) or $50 \mathrm{mg} / \mathrm{kg}$ i.p. CX-4945 twice daily $\mathrm{d} 7-12$ based on previous dose finding studies. BLI was performed at d7, d10, d13 and d15. At d10, d13 or d15 mice were anesthetized and euthanized by cervical dislocation. Leukemic blast frequency was analyzed in peripheral blood (PB), bone marrow (BM) and spleen by flow cytometry $\left(\mathrm{GFP}^{+}\right)$using FACSCalibur and CellQuest ${ }^{\mathrm{TM}}$ Pro software (BD, Heidelberg, Germany). Means and standard deviation of BLI and blast frequency values of all mice of a study group were calculated. Student's t-test was performed to compare study groups and values $<0.05$ were considered significant. Spleens, tibiae and femora were stored on ice in PBS (Biochrom, Berlin, Germany) containing 2\% FCS (Biochrom) until tumor cell isolation.

\section{ALL cell enrichment from spleen and bone marrow} Briefly, spleens were passed through $100 \mu \mathrm{m}$ cell strainers and incubated with erythrocyte lysis buffer (155 mM NH 4 Cl, 10 mM $\mathrm{KHCO}_{3}, 0.1 \mathrm{mM}$ EDTA). BM 
cells were isolated from tibiae and femora using PBS containing $2 \%$ FCS. Leukemic blast frequency was determined in BM and spleen cell populations by flow cytometry $\left(\mathrm{GFP}^{+}\right)$using FACSCalibur and CellQuest ${ }^{\mathrm{Tm}}$ Pro software (BD). For enrichment of human ALL cells, mouse cells were depleted using the Mouse Cell Depletion Kit (Miltenyi Biotec, Bergisch Gladbach, Germany) for use with AutoMACS (Miltenyi Biotec) according to the manufacturer's guidelines.

\section{Mass spectrometric analysis of CX-4945 in mice sera}

CX-4945 was determined by liquid coupled tandem mass spectrometry (LC-MS/MS) in the sera of treated mice to which the internal standard acridin orange was added prior to extraction. For this purpose, $10-250 \mu \mathrm{l}$ serum was extracted by triple liquid-liquid extraction with ethyl acetate and $n$-hexane $(1: 3 \mathrm{~V} / \mathrm{V})$. The first extraction was only ethyl acetate and the solvent was evaporated under nitrogen. The residue was then reconstituted in $100 \mu \mathrm{l}$ of a mixture of mobile phases A and $\mathrm{B}(65 \% \mathrm{~A})$ and $80 \mu \mathrm{l}$ were mass spectrometrically analyzed. Mass spectrometric analysis was performed on a Micromass Quattro Micro ${ }^{\text {Th }}$ API mass spectrometer. A HPLC Shimadzu LC-20 AD was used to separate the samples. The separation was performed using a Multospher $120 \mathrm{C} 18$ AQ column $125 \times 2 \mathrm{~mm}, 5 \mu \mathrm{m}$ particle size (CS-Chromatographie Service $\mathrm{GmbH}$, Langerwehe, Germany) coupled with a guard column $20 \times 3 \mathrm{~mm}$, $5 \mu \mathrm{m}$ particle size at a flow rate of $0.3 \mathrm{ml} / \mathrm{min}$. The mobile phase A consisted of water with $0.2 \%$ formic acid and the mobile phase $\mathrm{B}$ consisted of acetonitrile with $0.2 \%$ formic acid. CX-4945 was ionized by electrospray ionization and analyzed in positive mode using multiple reaction monitoring. The transitions were $\mathrm{m} / z 350.04$ (cone voltage $40 \mathrm{~V}$ ) to $\mathrm{m} / z 223.1$ with a collision energy of $12 \mathrm{~V}$ and $\mathrm{m} / z 314.1$ with a collision energy of $24 \mathrm{~V}$. The transitions $m / z 223.1$ was used as quantifier and $m /$ $z 314.1$ as qualifier. For the internal standard the following transitions were used: $m / z 266.11$ (cone voltage 24 V) to $m / z 234.1$ with $44 \mathrm{~V}$ collision energy and $m / z$ 250.1 with $24 \mathrm{~V}$. A standard solution curve was linear in a range of 5-100 nM. The concentration of CX-4945 in the sera of mice was obtained by extrapolation of the measured concentration to $1 \mathrm{ml}$.

\section{Custom panel targeted RNA sequencing}

RNA isolation of BM ALL cell fractions was performed using the AllPrep DNA/RNA/Protein Mini Kit (Qiagen, Hilden, Germany). Expression analyses were performed on the Ion Personal Genome Machine ${ }^{\mathrm{Tw}}\left(\mathrm{PGM}^{\mathrm{m}}\right)$ System (Ion Torrent ${ }^{\mathrm{TM}}$ ) (Thermo Fisher Scientific, Massachusetts, USA). For targeted RNA sequencing, an in-house custom panel was designed using Ion AmpliSeq Designer (Thermo Fisher Scientific), containing 179 genes involved in B-cell-receptor- and PI3K/AKT pathway signaling. Ion AmpliSeq RNA Libraries were prepared according to the manufacturer's protocol (MAN0006735). In brief, RNA was quantified with Qubit RNA HS Assay Kit (Thermo Fisher Scientific) and Qubit 2.0 Fluorometer (Life Technologies, CA, USA) and transcribed into cDNA by SuperScript VILO cDNA Synthesis Kit (Thermo Fisher Scientific). cDNA targets were amplified, amplicons partially digested, ligated to the adapters and purified with the Ion AmpliSeq ${ }^{\mathrm{mm}}$ Library Kit 2.0 (Thermo Fisher Scientific) in the ProFlex PCR System (Thermo Fisher Scientific). The final librabries were quantified by Ion Library TaqMan Quantification Kit (Thermo Fisher Scientific) using the ViiA 7 RealTime PCR System (Thermo Fisher Scientific). Following this, template preparation was carried out with the Ion PGM Hi-Q View OT2 Kit - 200 using the Ion One Touch 2 Instrument (Thermo Fisher Scientific) and enrichment of template-positive Ion Sphere Particles (ISP) with the Ion OneTouch ES (Thermo Fisher Scientific). The sequencing reaction run was performed with the PGM System and 400 flows. The evaluation of data sets was performed using Transcriptome Analysis Console (TAC) Software 4.0.0.25 (Thermo Fisher Scientific). The following filter criteria were set to identify relevantly regulated genes: $\operatorname{Avg}(\log 2)>5$, fold change $>2$ or $<-2$, $p$-value $<0.05$.

\section{Cell culture and inhibitor exposition}

Human B-ALL precursor cell lines SEM (ACC 546), RS4;11 (ACC 508) and NALM-6 (ACC 128) were purchased from German Collection of Microorganisms and Cell Cultures (DSMZ, Braunschweig, Germany) in 2004 (SEM and RS4;11) and 2016 (NALM-6), cultured as recommended by the manufacturer at $37^{\circ} \mathrm{C}$ and $5 \% \mathrm{CO}_{2}$. Media were supplemented with $10 \%$ heat-inactivated FCS (Biochrom, Berlin, Germany) and $100 \mu \mathrm{g} / \mathrm{ml}$ penicillin and streptomycin (Biochrom, Berlin, Germany). All cell lines were regularly tested for mycoplasma contamination (PCR) and authenticity (cell surface markers by flow cytometry) shortly before experiment initiation. $3.3 \times 10^{5}$ cells per ml were incubated with $5 \mu \mathrm{M} \mathrm{CX}$ 4945 (Hycultec, Beutelsbach, Germany) or DMSO (control) for up to $96 \mathrm{~h}$. Experiments were carried out in biological triplicates.

\section{Western blot}

Cells were lysed using RIPA buffer (Cell Signaling) and ultra sound exposure. Proteins $(30 \mu \mathrm{g})$ were separated on Midi gels (Bio-Rad, Munich, Germany), blotted onto a PVDF membrane (Bio-Rad) using the Trans-Blot ${ }^{\circ}$ Turbo $^{\text {ts }}$ Transfer System (Bio-Rad, 2.5 A, $25 \mathrm{~V}, 10 \mathrm{~min}$ ), blocked in LI-COR (Lincoln, NE, USA) blocking buffer and detected via LI-COR Odyssey Imaging System and 
Image Studio Lite software. The following antibodies were used for protein detection: pAKT (Ser473), AKT, BCL6 (clone D4I2V), BACH2 (clone D3T3G; all Cell Signaling), GAPDH (clone ZG003; Invitrogen), IRDye 680RD Goat anti-Mouse, IRDye $800 \mathrm{CW}$ Goat antiMouse, IRDye 680RD Goat anti-Rabbit and IRDye 800CW Goat anti-Rabbit (all LI-COR). Experiments were carried out in biological triplicates.

\section{$B C L 6$ and $B A C H 2$ gene expression analysis}

RNA isolation of BM tumor cell fractions and cell culture samples was performed using the AllPrep DNA/RNA/ Protein Mini Kit (Qiagen, Hilden, Germany) and miRNeasy $^{\oplus}$ Mini Kit (Qiagen), respectively, and followed by cDNA synthesis using the PrimeScript ${ }^{\mathrm{Tm}} \mathrm{RT}$ Reagent Kit (Takara Bio Europe, Saint-Germain-en-Laye, France) according to the manufacturers' protocols. Gene expression analysis was carried out in technical triplicates using TB Green $^{\mathrm{TM}}$ Premix Ex Taq $^{\mathrm{Tm}}$ II mastermix (Takara Bio Europe) with $0.3 \mu \mathrm{M}$ primers (BCL6 forward CTGTGATGGC CACGGCTAT; BCL6 reverse CGGCAAGTGTCCACAA; $B A C H 2$ forward GCGGCCCCAAATTAAATGT; $B A C H 2$ reverse AACGATCCGGATTCGTCACT; GAPDH forward CTGCACCACCAACTGCTTAG; GAPDH reverse GTCTTCTGGGTGGCAGTGAT) and $10 \mathrm{ng}$ cDNA in a ViiA7 Real Time PCR system (Applied Biosystems, Foster City, CA, USA) using the following protocol: $15 \mathrm{~min}$ initial denaturation at $95^{\circ} \mathrm{C}$ followed by 45 cycles of $15 \mathrm{~s}$ denaturation at $94{ }^{\circ} \mathrm{C}, 30 \mathrm{~s}$ annealing at $54^{\circ} \mathrm{C}$ and $30 \mathrm{~s}$ elongation at $72{ }^{\circ} \mathrm{C}$. Relative gene expression was normalized to GAPDH and calculated using the $2^{-\Delta \Delta \mathrm{Ct}}$ formula. Cell culture experiments were carried out in biological triplicates. Four mice per group and time point were analyzed. Results are described as mean \pm standard deviation. Significance $(p<0.05)$ was estimated by 2 -tailed student's t test.

\section{Results \\ Early effects of targeted CK2 inhibition on B-ALL xenograft mice}

Previous studies involving CX-4945 anti-tumor regimens are mainly based on observations made at time points several days after the last therapeutic dose. We herein focused on CX-4945-mediated effects during and shortly after therapy. To evaluate the immediate effect of CK2 inhibition (twice daily from d7 to d13) on the proliferation of SEM cells in NSG mice, animals were sacrificed during (d10), immediately after (d13) or $48 \mathrm{~h}$ after the last therapeutic dose (d15). To examine tumor cell proliferation and distribution, longitudinal bioluminescence imaging (BLI) of all animals was performed on d7, d10, $\mathrm{d} 13$ and d15. No differences in blast distribution (Fig. 1a) and proliferation (Fig. 1b) were observed between treated and untreated animals.
Pharmacokinetic experiments were conducted to elucidate in which concentrations CX-4945 was present in serum of treated mice (Fig. 1c). During therapy CX-4945 concentrations of $190 \pm 150 \mu \mathrm{M}(\mathrm{d} 10)$ and $155 \pm 88 \mu \mathrm{M}$ (d13) were measured. CX-4945 levels then declined rapidly: two days after the final CX-4945 application (d15) traces were detectable only in one out of four mice. No traces of CX-4945 were detected in untreated animals.

Investigations of cells and tissues revealed only minimal changes. BM tumor cell frequency determined by flow cytometry was lower in CX-4945-treated animals compared to controls at all time points (Fig. 1d). Tumor cell frequency in control animals increased rapidly from $3.3 \pm 1.4 \%$ (d10) to $18.4 \pm 8.5 \%$ (d15) while CX-4945treated mice showed decelerated blast counts $(2.7 \pm$ $1.2 \%, 4.7 \pm 1.3 \%, 9.2 \pm 8.5 \%$ at $\mathrm{d} 10, \mathrm{~d} 13$, d15, respectively). Spleen infiltration of ALL cells was reduced accordingly (Fig. 1e; Control: $2.3 \pm 3.1 \%$ vs CX-4945: $1.1 \pm$ $0.4 \%$ at $\mathrm{d} 15)$.

Further, BLI was performed for several organs to identify minor tumor cell infiltration (Additional File 2: Fig. S1). Blasts were mostly localized in tibiae, femora and skull while infiltration of sternum, spleen, liver and lung was rarely observed. Tumor cell distribution was comparable in controls and CX-4945-treated animals.

\section{Downregulation of $B C L 6$ induces apoptosis in BM-derived ALL blasts}

To identify molecular pathways involved in the decreased BM and spleen blast infiltration, we next performed gene expression profiling. We analyzed 177 genes involved in $\mathrm{B}$ cell receptor (BCR), PI3K/AKT and CK2 downstream signaling in selected bone marrow blast samples (Additional File 1: Table S2). Samples taken at the distinct observation time points (d10, d13, d15) were analyzed separately, revealing significant upregulation of CDC42, CD19 and JAK1 after CX-4945 therapy at $\mathrm{d} 10$ (Fold changes 2.22, 2.71 and 2.29 compared to controls, respectively). However, these changes were not found at $\mathrm{d} 13$ and $\mathrm{d} 15$. In fact, no relevant upor downregulation was present at the latter time points. Comparing all controls and treated animals, no relevant difference was observed and groups did not form distinct clusters (Fig. 2a, b).

Throughout all time points, gene expression of antiapoptotic transcription factor BCL6 was slightly reduced after CX-4945 therapy (Fold changes $-1.17,-1.15$ and - 1.11 compared to controls at $\mathrm{d} 10, \mathrm{~d} 13$ and $\mathrm{d} 15$, respectively). In contrast, expression of BCL2L11, an inducer of apoptosis, was increased (Fold changes 1.76, 1.04, 1.40; Additional File 3: Table S2), possibly encouraging pro-apoptotic signaling (Fig. 2c). Interestingly, apoptosis-inducing $B A D, I K B K B$ and $R E L A$ expression was decreased, suggesting the previously observed 


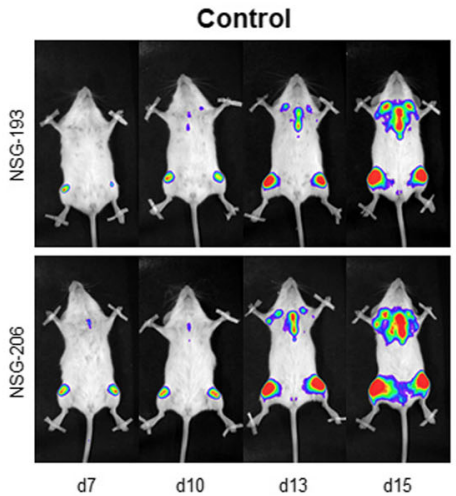

b

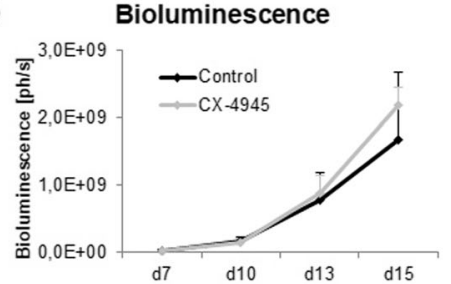

d

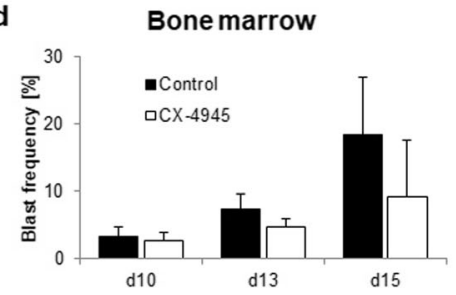

CX-4945
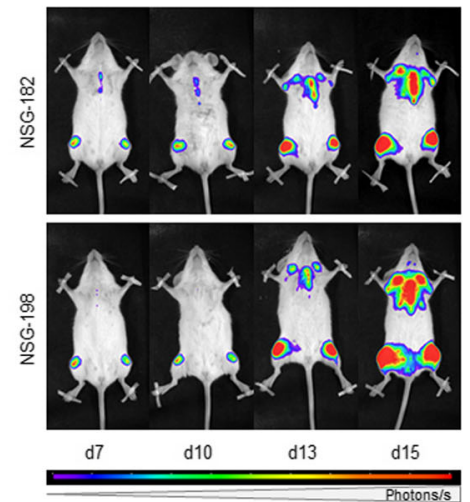

c CX-4945 serum concentration

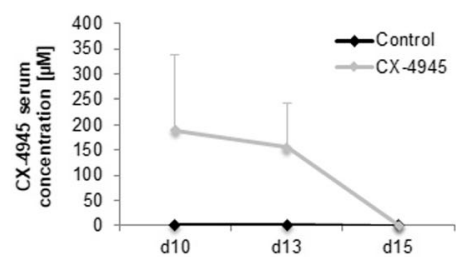

e

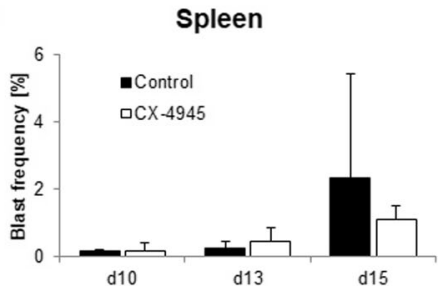

Fig. 1 Evaluation of CX-4945 application on NSG mice engrafted with SEM B-ALL cells. NSG mice were i.v.-injected with $2.5 \times 10^{6}$ GFP-and luciferase-transduced SEM cells and treated with vehicle (control) or $50 \mathrm{mg} / \mathrm{kg}$ CX-4945 i.p. twice daily from d7-13. Mice were sacrificed on d10, d13 or d15 for subsequent analyses. a Representative images of longitudinal bioluminescence imaging of two control animals and two CX-4945treated animals in dorsal position. $\mathbf{b}$ Longitudinal bioluminescence imaging was conducted at $\mathrm{d} 7$ to verify tumor cell engraftment and subsequently repeated at all study days. Increasing luminescence is proportional to proliferation of luciferase-expressing blasts. Quantification of full body bioluminescence (ph/s) after treatment was performed by adding total luminescence signals of dorsal and ventral imaging. Four animals per time point and study group; mean \pm standard deviation. c Pharmacokinetic analyses were conducted at d10, d13 and d15 to investigate CX-4945 serum concentrations. Serum levels were determined by liquid coupled tandem mass spectrometry. Four animals per time point and study group; mean \pm standard deviation. $\mathbf{d}$,e Influence of CX-4945 on blast frequency in bone marrow $\mathbf{d}$ and spleen e. Tumor cell frequency was evaluated by flow cytometry of $\mathrm{GFP}^{+}$leukemic blasts. Four animals per time point and study group; mean \pm standard deviation

induction of cell death [10] was probably not mediated through NFkB. According to wikipathways, 15 genes included in our panel are involved in apoptotic signaling [20]. None of these was significantly regulated by CX4945 therapy at any time point. Most genes showed ambiguous gene expression profiles during observation time points. Raw data, fold changes and $p$-values of all genes for respective time points are summarized in Additional File 4: Table S3.

\section{CX-4945-induced apoptosis is evoked by BCL6 deregulation}

As BCL6 was found consistently downregulated in RNA sequencing analyses and can be regulated by CX-4945 target Ikaros, we evaluated this pathway in more detail.
We also investigated BCL6 counterpart and tumor suppressor BACH2.

For characterization of $\mathrm{BCL} 6$ and $\mathrm{BACH} 2$ involvement in anti-proliferative effects after CK2 inhibition, MLLrearranged pro-B-ALL cell lines SEM and RS4;11 as well as $\mathrm{BCR}^{+}$pre-B-ALL cell line NALM- 6 were incubated with CX-4945 for up to $96 \mathrm{~h}$. All cell lines initially expressed different amounts of $\mathrm{BACH} 2$ and BCL6 proteins (Additional File 5: Fig. S2). High levels of BCL6 correlated with low BACH2 expression (SEM, RS4;11) and vice versa (NALM-6).

In SEM cells, $B A C H 2$ gene expression (Fig. 3a) and protein expression (Fig. 3b, c) remained constant during short term-incubation $(<24 \mathrm{~h})$ while incubation for extended periods induced decreased gene and protein 
a

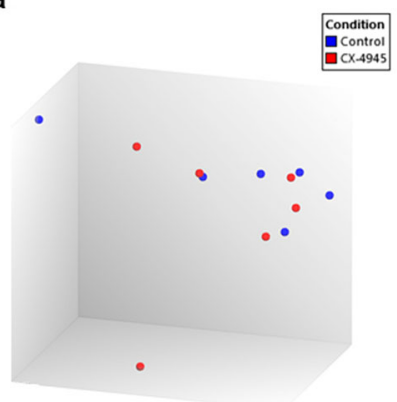

b

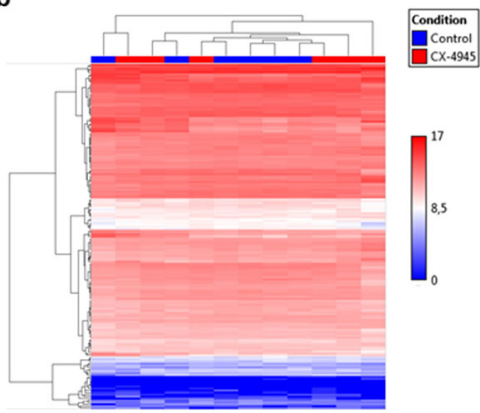

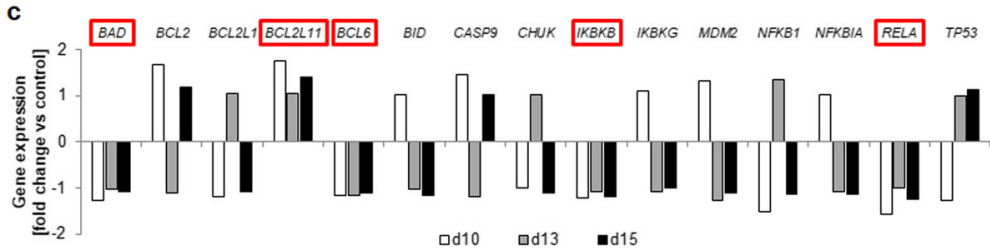

Fig. 2 Custom panel targeted RNA sequencing of treated and untreated mice. Bone marrow-derived leukemic blast populations of controls and treated mice sacrificed at d10, d13 and d15 (two mice per group and time point) were analyzed for changes in gene expression of 177 genes involved in BCR, PI3K/AKT and CK2 downstream signaling. a Principal component analysis blot of control animals (blue) and CX-4945-treated animals (red) demonstrating no distinct clustering of subgroups. b Hierarchical clustering of all genes and all animals throughout all observation time points. c Fold changes of apoptosis-related genes within the panel after CX-4945 treatment compared to time-matched controls. No significant regulation (fold change $>2$ or $<-2$ ) was observed. Red boxes indicate genes with consistent gene expression changes throughout all time points

BACH2

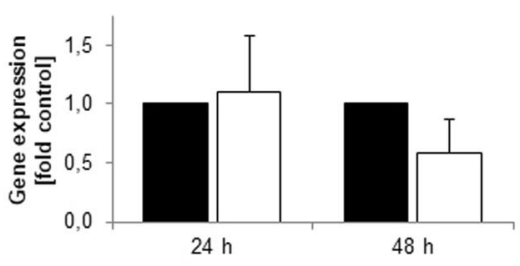

b

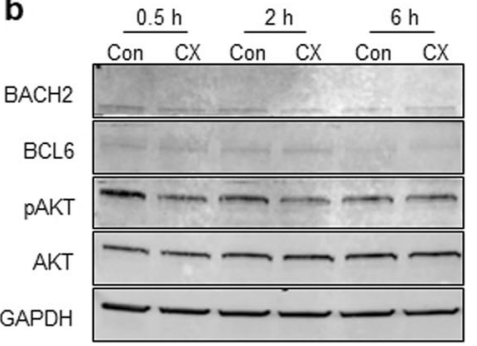

c

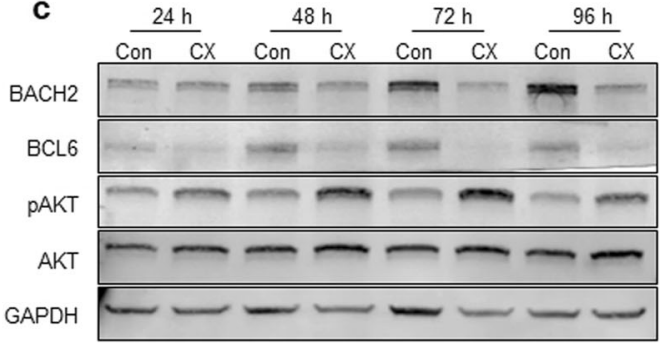

Fig. 3 Evaluation of CX-4945-induced effects on BACH2 and BCL6 gene and protein expression in SEM cells. SEM cells were cultured and incubated with $5 \mu \mathrm{M}$ CX-4945 or DMSO (control) for up to $96 \mathrm{~h}$. a Changes in BACH2 and BCL6 gene expression were assessed by qRT-PCR after $48 \mathrm{~h}$ and $72 \mathrm{~h}$. Mean values of $\Delta C T$ values from DMSO-treated control cells were calculated and set to 1 for each time point. $\Delta \Delta C T$ values were calculated for CX-4945-treated samples and compared to the respective time-matched control. Analyses were carried out in three independent biological and technical replicates. Mean \pm standard deviation; * $p<0.05$. b, c Analysis of BACH2 and BCL6 protein expression as well as AKT phosphorylation was carried out by western blot with GAPDH as housekeeping gene. Representative images of three independent biological experiments. Blots were processed and cropped using Image Studio Lite 5.2 software and MS PowerPoint (2011) to improve clarity and conciseness. Full size blots are uploaded in Additional File 8: Fig. S5. b Short term effects of CX-4945 incubation were determined after $0.5 \mathrm{~h}, 2 \mathrm{~h}$ and 6 h. c Long term effects of CX-4945 incubation were determined after 24 h, 48 h, $72 \mathrm{~h}$ and $96 \mathrm{~h}$ 
expression. Similar results were observed for BCL6 with significantly reduced gene expression levels after $48 \mathrm{~h}$ (0.31-fold, $p=0.011 ; 24$ h: 0.55 -fold, $p=0.053$ ). BCL6 protein levels also decreased after $24 \mathrm{~h}, 48 \mathrm{~h}, 72 \mathrm{~h}$ and $96 \mathrm{~h}$ of CX-4945 incubation.

Phosphorylation of AKT residue Ser473 was evaluated to further characterize the effect of CX-4945 on PI3K signaling. AKT phosphorylation decreased after short term incubation with CX-4945 (Fig. 3b). Conversely, pAKT levels were elevated after extended incubation periods (Fig. 3c). Total AKT protein expression remained constant throughout all time points observed.

To further validate the observed effects, the influence of CX-4945 on B-ALL cell lines of distinct molecular backgrounds (RS4;11, NALM-6) was determined on protein level. Both cell lines exhibited reduced $\mathrm{BACH} 2$ and BCL6 expression detectable from as early as $6 \mathrm{~h}$ incubation (Additional File 6: Fig. S3). In RS4;11 cells, pAKT levels decreased during short term incubation (Additional File 6: Fig. S3a) before increasing after $48 \mathrm{~h}, 72 \mathrm{~h}$ and $96 \mathrm{~h} \mathrm{CX}-4945$ incubation compared to DMSOtreated control cells (Additional File 6: Fig. S3b). Even though no basal AKT phosphorylation was detected in NALM-6 cells, pAKT levels increased after extended incubation with CX-4945 (Additional File 6: Fig. S3d).

For comparison with $B C L 6$ and in vitro data, gene expression analysis of BM-derived tumor cell populations was subsequently performed for $B A C H 2$ (Additional File 7: Fig. S4). $B A C H 2$ gene expression was slightly increased at d10 and d15 (1.19-fold and 1.26-fold compared to control animals, respectively). Though, changes were not significant $(p=0.234$ and 0.333 , respectively), matching our findings that changes in BCL6 rather than $B A C H 2$ gene expression contribute to altered apoptotic signaling.

\section{Discussion}

CK2 is frequently upregulated in many neoplasms including B-ALL [4]. The selective CK2 inhibitor CX-4945 has previously been shown to exhibit anti-proliferative effects in vitro and heterogeneously in vivo $[10,11,15$, 17, 21-23]. However, molecular mechanisms and the exact mode of action of CX-4945 in B-ALL remain widely unknown. We have previously demonstrated induction of apoptosis in B-ALL cell lines including SEM [10]. Other groups also described CX-4945-mediated apoptosis in various tumor cells including B-ALL $[8,11$, $13,15,17,22,24-27]$. Thus, the aim of the current study was to evaluate whether CX-4945 treatment of BALL xenograft mice results in early reduced proliferation. Further, we investigated CX-4945-induced apoptotic and molecular processes during and after CK2 inhibition using in vitro and in vivo approaches. Our results suggest that downregulation of the oncogenic transcription factor BCL6 might contribute to antiproliferative signaling.

While previous in vitro experiments using CX-4945 demonstrated significant anti-proliferative effects in BALL cell lines, our results could not demonstrate this effect in a B-ALL xenograft model. In contrast, Song et al. detected prolonged survival and reduced tumor cell proliferation in B-ALL engrafted mice [28]. This discrepancy might be due to different cell lines used or varying therapeutic regimens. Our study used lower CX-4945 doses and shorter application periods than Song et al. Also, we evaluated tumor cell proliferation during or immediately after our short therapeutic period, explaining the lack of anti-proliferative action. In line with our observations, Prins et al. reported only minimal effects of CX-4945 on de novo B-ALL xenograft mice [22]. To ensure that the lack of anti-proliferative effects was not due to limited bioavailability, we performed pharmacokinetic studies and demonstrated that CX-4945 was present in blood serum samples of treated animals. Achieved CX-4945 concentrations are similar to otherwise reported effective concentrations. Serum levels declined rapidly when the drug was removed, which is in line with the observations of Siddiqui-Jain et al. who also reported a quick reduction of plasma concentrations from as early as $15 \mathrm{~min}$ after CX-4945 removal [13]. Other groups calculated a CX-4945 half-life of $\sim 5 \mathrm{~h}$ in mice [29]. This rapid decline in CX-4945 bioavailability might explain the continued proliferation of B-ALL tumor cells after therapy we observed in our previous study. This finding suggests that prolonged or continuous CX-4945 exposure might overcome the limited therapeutic potential [10].

Still, in our present cohort CX-4945 treatment of mice engrafted with B-ALL cell line SEM resulted in decreased blast frequencies in BM and spleen. This indicates that even short application periods and a moderate dosis of CX-4945 are sufficient to induce signaling-modulating changes in tumor cells. Focusing on BCR- and PI3K/ AKT-related genes, we performed targeted RNA sequencing to firstly evaluate if PI3K downstream signaling modifications could be responsible for the observed effects. Analysis of $\mathrm{BM}$-derived blasts revealed a significant upregulation of CDC42, CD19 and JAK1 after CX-4945 treatment. JAK1 activation and $C D 19$ overexpression are generally not associated with anti-leukemic signaling but with leukemogenesis $[30,31]$. In contrast, the small GTPase CDC42 plays a key role in cell cycle regulation [32] and might be involved in apoptotic signaling via activation of JNK and FasL in HL-60 and Jurkat cells [33, 34].

We then evaluated the gene expression of apoptosisrelated genes after CX-4945 incubation in more detail. We found that the anti-apoptotic transcription factor $B C L 6$ was constantly, yet not significantly, 
downregulated throughout all observation time points. Most other genes involved in apoptosis showed ambiguous regulation patterns. In line with this finding, $\mathrm{Ge}$ et al. recently reported that in vitro BCL6 gene expression is regulated by direct CX-4945 target Ikaros, and that increased BCL6 expression in adult B-ALL patients is associated with inferior outcomes [35]. We subsequently evaluated protein expression of BCL6 and its opponent, tumor-suppressing transcription factor $\mathrm{BACH} 2$ in B-ALL cell lines after CX-4945 incubation and found reduced BCL6 levels. Unexpectedly and in contrast to Ge et al., $\mathrm{BACH} 2$ gene and protein levels were also decreased. This could be explained by CX-4945-mediated regulatory mechanisms other than Ikaros binding, or by upstream signaling modulation in the cell lines used. $B A C H 2$ gene expression can be controlled by hypoxiainduced factor $1 \alpha$, a target protein of CK2 [36-38]. Also, Tamahara et al. further demonstrated AKT- and mTORmediated inhibition of gene and protein expression of $\mathrm{BACH} 2$ [39]. This matches our findings of both decreased BACH2 levels as well as AKT activation occurring after extended CX-4945 incubation periods. This observation suggests the possibility of a shared AKT and BACH2 regulatory mechanism.

In vitro western blot analyses in SEM and RS4;11 cells demonstrated that the direct CK2 target protein AKT was initially dephosphorylated by CK2 inhibition. This results in decreased PI3K/AKT pathway activity and potential induction of apoptotic signaling via downstream mechanisms. Unexpectedly, further CX-4945 incubation evoked strong phosphorylation of AKT even in the initially phospho-AKT-negative cell line NALM-6, suggesting an induction of cellular escape mechanisms to evade apoptosis. The analyzed AKT residue Ser473 is phosphorylated by PI3K/AKT pathway member mTOR and necessary for AKT kinase activity, underlining the involvement of this signaling cascade in CX-4945mediated anti-leukemic effects [40]. Recently Chen et al. as well as Baumgarten et al. both reported feedback loops between AKT and MEK signaling, suggesting increased AKT phosphorylation might be evoked by escape strategies involving upregulation of the MEK pathway $[41,42]$. This also fits in well with the increased gene expression of CDC42 observed after CX-4945 treatment, with CDC42 being involved in JNK and p38 signaling cascades [43]. However, whole transcriptome and whole methylome sequencing approaches as well as further pharmacokinetic experiments are necessary to elucidate in detail how CX-4945 influences distinct signaling cascades. In addition, combinatory approaches using mTOR, AKT and MEK inhibitors can shed light on so far unexplored pathway regulation and feedback loops. Also, these results should be validated in human primary B-ALL cells.

\section{Conclusions}

In conclusion, we herein identify a potential mechanism for the regulation of apoptotic processes in B-ALL in vitro and in vivo. Apoptosis is probably evoked by Ikaros-mediated downregulation of BCL6 and tightly regulated by AKT. Limitations in anti-proliferative and pro-apoptotic CX-4945-induced effects could possibly be overcome by additional application of AKT or MEK inhibitors.

\section{Supplementary information}

Supplementary information accompanies this paper at https://doi.org/10. 1186/s12885-020-6650-9.

\begin{abstract}
Additional File 1: Table S1. List of all mice recruited in the study, respective end points and analyses conducted.

Additional File 2: Figure S1. Evaluation of CX-4945 application on organ infiltration in SEM-engrafted NSG mice. NSG mice were i.v.-injected with $2.5 \times 10^{6}$ GFP- and luciferase-transduced SEM cells and treated with vehicle (control) or $50 \mathrm{mg} / \mathrm{kg}$ CX-4945 i.p. twice daily from d7-13. Mice were sacrificed on d10, d13 or d15 for subsequent analyses. Bioluminescence imaging of brain, skull, lung, heart, liver, sternum, spleen, kidney, femur, tibia and fat tissue was performed directly after mice were sacrificed. Four animals per time point and study group.
\end{abstract}

Additional File 3: Table S2. Gene expression fold changes of genes involved in apoptotic processes.

Additional File 4: Table S3. Gene expression fold changes and $p$ values of all genes included in the BCR/PI3K/CK2 panel.

Additional File 5: Figure S2. Basal characterization of protein expression in B-ALL cell lines SEM, RS4;11 and NALM-6. Analysis of BACH2 and BCL6 protein expression was carried out by western blot with GAPDH as housekeeping gene. Representative images of three independent biological experiments. Blots were processed and cropped using Image Studio Lite 5.2 software and MS PowerPoint (2011) to improve clarity and conciseness. Full size blots are uploaded in Additional File 8: Fig. S5.

Additional File 6: Figure S3. Evaluation of AKT phosphorylation, $\mathrm{BACH} 2$ and $\mathrm{BCL} 6$ protein expression in RS4;11 and NALM-6 cells. RS4;11 $(\mathbf{a}, \mathbf{b})$ and NALM-6 cells (c, d) were cultured and incubated with $5 \mu \mathrm{M}$ CX-4945 or DMSO (control) for up to $96 \mathrm{~h}$. Analysis of BACH2 and BCL6 protein expression as well as AKT phosphorylation was carried out by western blot with GAPDH as housekeeping gene. Representative images of three independent biological experiments. Short term effects of CX4945 incubation were determined after $0.5 \mathrm{~h}, 2 \mathrm{~h}$ and $6 \mathrm{~h}(\mathbf{a}, \mathbf{c})$. Long term effects of CX-4945 incubation were determined after 24Blots were processed and cropped using Image Studio Lite 5.2 software and MS PowerPoint (2011) to improve clarity and conciseness. Full size blots are uploaded in Additional File 8: Fig. S5.

Additional File 7: Figure S4. Gene expression analysis of $B A C H 2$. Bone marrow-derived leukemic blast populations of controls and treated mice sacrificed at d10, d13 and d15 were analyzed for changes in gene expression of $B A C H 2$ using $\mathrm{qPCR}$. Mean values of $\triangle C T$ values from controls were calculated and set to 1 for each time point. $\Delta \Delta C T$ values were calculated for CX-4945-treated samples and compared to the respective timematched control. Analyses were carried out in three technical replicates. Four animals per time point and study group; mean \pm standard deviation.

Additional File 8: Figure S5. Original blots for Fig. 3b, c, S2, S3. Red boxes indicate the regions used in the respective Figures.

\section{Abbreviations}

AML: Acute myeloid leukemia; B-ALL: Acute B-lymphoblastic leukemia; BCR: B cell receptor; BLI: Bioluminescence imaging; BM: Bone marrow; CK2: Casein kinase II; Ffluc: Firefly luciferase; LC-MS/MS: Liquid phase coupled tandem mass spectrometry; PB: Peripheral blood 


\section{Acknowledgements}

We would like to thank Irmela Jeremias and Binje Vick for lentiviral transduction of ALL cell lines and excellent collaboration.

\section{Authors' contributions}

AR participated in study design, performed in vivo and in vitro experiments as well as data analysis, wrote the manuscript. SS performed RNA sequencing experiments, participated in RNA sequencing analysis, participated in writing the manuscript. AL performed in vitro experiments and data analysis. RS performed pharmacokinetic analyses and data interpretation. GK provided technical assistance for in vivo experiments. AS provided technical assistance for in vitro experiments. BH revised manuscript. HME critically revised manuscript. CJ critically revised manuscript. CR designed study, established xenograft model, participated in data analysis and interpretation, critically revised manuscript. All authors approved the final version of the paper.

\section{Funding}

AR received a scholarship from the German federal state of MecklenburgVorpommern. CR received a FORUN grant from the University of Rostock. The funders had no role in study design, data collection, analysis and interpretation, decision to publish, or preparation of the manuscript.

\section{Availability of data and materials}

The datasets used and/or analysed during the current study are available from the corresponding author on reasonable request.

\section{Ethics approval and consent to participate}

The study was performed in accordance to the Declaration of Helsinki and the local ethical standards of the Rostock University Medical Center. All animal experiments were approved by the review board of the federal state of Mecklenburg-Vorpommern, Germany (reference number: LALLF MV/ 7221.3-1.1-002/15). None of the cell lines used need ethics approval.

\section{Consent for publication}

Not applicable.

\section{Competing interests}

The authors declare that they have no competing interests.

\section{Author details}

'Department of Medicine, Clinic III - Hematology, Oncology, Palliative Medicine, Rostock University Medical Center, Ernst-Heydemann-Strasse 6, 18057 Rostock, Germany. ${ }^{2}$ Institute of Pharmacology and Toxicology, Rostock University Medical Center, Schillingallee 70, 18057 Rostock, Germany.

Received: 14 November 2019 Accepted: 17 February 2020

Published online: 04 March 2020

\section{References}

1. Chua M, Ortega C, Sheikh A, Lee M, Abdul-Rassoul H, Hartshorn K, Dominguez I. CK2 in Cancer: cellular and biochemical mechanisms and potential therapeutic target. Pharmaceuticals. 2017;10:18

2. Rabalski AJ, Gyenis L, Litchfield DW. Molecular pathways: emergence of protein kinase CK2 (CSNK2) as a potential target to inhibit survival and DNA damage response and repair pathways in Cancer cells. Clin Cancer Res. 2016;22:2840-7.

3. Nuñez de Villavicencio-Diaz T, Rabalski A, Litchfield D. Protein Kinase CK2: Intricate Relationships within Regulatory Cellular Networks. Pharmaceuticals. 2017;10:27.

4. Buontempo F, McCubrey JA, Orsini E, Ruzzene M, Cappellini A, Lonetti A, Evangelisti C, Chiarini F, Evangelisti C, Barata JT, Martelli AM. Therapeutic targeting of CK2 in acute and chronic leukemias. Leukemia. 2018;32:1-10.

5. Duncan JS, Turowec JP, Duncan KE, Vilk G, Wu C, Lüscher B, Li SSC, Gloor $G B$, Litchfield DW. A peptide-based target screen implicates the protein kinase CK2 in the global regulation of caspase signaling. Sci Signal. 2011;4: ra30.

6. Eddy SF, Guo S, Demicco EG, Romieu-Mourez R, Landesman-Bollag E, Seldin DC, Sonenshein GE. Inducible IkappaB kinase/lkappaB kinase epsilon expression is induced by CK2 and promotes aberrant nuclear factor-kappaB activation in breast cancer cells. Cancer Res. 2005;65:11375-83.
7. Cheong J-W, Min YH, Eom JI, Kim SJ, Jeung HK, Kim JS. Inhibition of CK2\{alpha\} and PI3K/Akt synergistically induces apoptosis of CD34+CD38leukaemia cells while sparing haematopoietic stem cells. Anticancer Res. 2010;30:4625-34.

8. Quotti Tubi L, Canovas Nunes S, Brancalion A, Doriguzzi Breatta E, Manni S, Mandato E, Zaffino F, Macaccaro P, Carrino M, Gianesin K, Trentin L, Binotto G, Zambello R, Semenzato G, Gurrieri C, Piazza F. Protein kinase CK2 regulates AKT, NF-KB and STAT3 activation, stem cell viability and proliferation in acute myeloid leukemia. Leukemia. 2017;31:292-300,

9. Chon HJ, Bae KJ, Lee Y, Kim J. The casein kinase 2 inhibitor, CX-4945, as an anti-cancer drug in treatment of human hematological malignancies. Front Pharmacol. 2015;6:70.

10. Richter A, Roolf C, Hamed M, Gladbach YS, Sender S, Konkolefski C, Knübel G, Sekora A, Fuellen G, Vollmar B, Murua Escobar H, Junghanss C. Combined casein kinase II inhibition and epigenetic modulation in acute Blymphoblastic leukemia. BMC Cancer. 2019;19(1):202.

11. Gomes AM, Soares MVD, Ribeiro P, Caldas J, Póvoa V, Martins LR, Melão A, Serra-Caetano A, de Sousa AB, Lacerda JF, Barata JT. Adult B-cell acute lymphoblastic leukemia cells display decreased PTEN activity and constitutive hyperactivation of PI3K/Akt pathway despite high PTEN protein levels. Haematologica. 2014;99:1062-8.

12. Siddiqui-Jain A, Bliesath J, Macalino D, Omori M, Huser N, Streiner N, Ho CB, Anderes K, Proffitt C, O'Brien SE, JKC L, Von HDD, Ryckman DM, Rice WG, Drygin D, Helleday T, Petermann E, Lundin C, Hodgson B, Sharma R, Guerra B, Issinger O, Tawfic S, Yu S, Wang H, Faust R, Davis A, Ahmed K, Loizou J, El-Khamisy S, et al. CK2 Inhibitor CX-4945 Suppresses DNA Repair Response Triggered by DNA-Targeted Anticancer Drugs and Augments Efficacy: Mechanistic Rationale for Drug Combination Therapy. Mol Cancer Ther. 2012;11:193-204.

13. Siddiqui-Jain A, Drygin D, Streiner N, Chua P, Pierre F, O'Brien SE, Bliesath J, Omori M, Huser N, Ho C, Proffitt C, Schwaebe MK, Ryckman DM, Rice WG, Anderes K, Luo J, Solimini N, Elledge S, Hanahan D, Weinberg R, Zhang J, Yang P, Gray N, Solimini N, Luo J, Elledge S, Ruzzene M, Pinna L, Guerra B, Issinger O, et al. CX-4945, an orally bioavailable selective inhibitor of protein kinase CK2, inhibits prosurvival and angiogenic signaling and exhibits antitumor efficacy. Cancer Res. 2010;70:10288-98.

14. Kim H, Choi K, Kang H, Lee SY, Chi SW, Lee MS, Song J, Im D, Choi Y, Cho S. Identification of a novel function of CX-4945 as a splicing regulator. PLOS One. 2014;9:1-8.

15. Buontempo F, Orsini E, Martins LR, Antunes I, Lonetti A, Chiarini F, Tabellini G, Evangelisti C, Melchionda F, Pession A, Bertaina A, Locatelli F, McCubrey JA, Cappellini A, Barata JT, Martelli AM. Cytotoxic activity of the casein kinase 2 inhibitor CX-4945 against T-cell acute lymphoblastic leukemia: targeting the unfolded protein response signaling. Leukemia. 2014;28:543-53.

16. Deplus R, Blanchon L, Rajavelu A, Boukaba A, Defrance M, Luciani J, Rothé F, Dedeurwaerder S, Denis H, Brinkman AB, Simmer F, Müller F, Bertin B, Berdasco M, Putmans P, Calonne E, Litchfield DW, de Launoit Y, Jurkowski TP, Stunnenberg HG, Bock C, Sotiriou C, Fraga MF, Esteller M, Jeltsch A, Fuks F. Regulation of DNA methylation patterns by CK2-mediated phosphorylation of Dnmt3a. Cell Rep. 2014;8:743-53.

17. Buontempo F, Orsini E, Lonetti A, Cappellini A, Chiarini F, Evangelisti C, Evangelisti C, Melchionda F, Pession A, Bertaina A, Locatelli F, Bertacchini J, Neri LM, McCubrey JA, Martelli AM. Synergistic cytotoxic effects of bortezomib and CK2 inhibitor CX-4945 in acute lymphoblastic leukemia: turning off the prosurvival ER chaperone BIP/Grp78 and turning on the proapoptotic NF-KB. Oncotarget. 2016;7:1323-40.

18. Roolf C, Richter A, Konkolefski C, Knuebel G, Sekora A, Krohn S, Stenzel J, Krause BJ, Vollmar B, Murua Escobar H, Junghanss C. Decitabine demonstrates antileukemic activity in B cell precursor acute lymphoblastic leukemia with MLL rearrangements. J Hematol Oncol. 2018;11:62.

19. Terziyska N, Castro Alves C, Groiss V, Schneider K, Farkasova K, Ogris M, Wagner E, Ehrhardt H, Brentjens RJ, zur Stadt U, Horstmann M, QuintanillaMartinez $\mathrm{L}$, Jeremias I. In vivo imaging enables high resolution preclinical trials on patients' leukemia cells growing in mice. PLoS One. 2012;7:52798.

20. Zambon AC, Hanspers K, Pico A, Kutmon M, Kalafati M, van lersel M, Riutta A, Willighagen E, Anam A, Lawlor B, Bouwman J, Josip MG, Coort S, Kelder T: Apoptosis (Homo sapiens). https://www.wikipathways.org/index.php/ Pathway:WP254 2019.

21. Martins LR, Mel A, Antunes I, Cardoso BA, Stansfield R, MT SB, Ghia P, Drygin $D$, Silva MG, Barata JT. Activity of the clinical-stage CK2-specific inhibitor CX4945 against chronic lymphocytic leukemia. Leukemia. 2013;28:179-82. 
22. Prins RC, Burke RT, Tyner JW, Druker BJ, Loriaux MM, Spurgeon SE. CX-4945, a selective inhibitor of casein kinase-2 (CK2), exhibits anti-tumor activity in hematologic malignancies including enhanced activity in chronic lymphocytic leukemia when combined with fludarabine and inhibitors of the B-cell receptor pathway. Leukemia. 2013;27:2094-6.

23. Martins LR, Lúcio P, Melao A, Cardoso BA, Stansfield R, Drygin D, Silva MG, Barata JT. In vitro and in vivo targeting of chronic lymphocytic leukemia using CX-4945, a clinical-stage CK2-specific inhibitor. Blood. 2012;120:2897.

24. Kendall JJ, Chaney KE, Patel AV, Rizvi TA, Largaespada DA, Ratner N, Kendall JJ, Chaney KE, Patel AV, Rizvi TA, Largaespada DA, Ratner N. CK2 blockade causes MPNST cell apoptosis and promotes degradation of $\beta$-catenin. Oncotarget. 2016;7:53191-203.

25. Lertsuwan J, Lertsuwan K, Sawasdichai A, Tasnawijitwong N, Lee K, Kitchen P, Afford S, Gaston K, Jayaraman P-S, Satayavivad J. CX-4945 induces Methuosis in Cholangiocarcinoma cell lines by a CK2-independent mechanism. Cancers (Basel). 2018;10:283.

26. Bliesath J, Huser N, Omori M, Bunag D, Proffitt C, Streiner N, Ho C, SiddiquiJain A, O'Brien SE, Lim JKC, Ryckman DM, Anderes K, Rice WG, Drygin D. Combined inhibition of EGFR and CK2 augments the attenuation of PI3KAkt-mTOR signaling and the killing of cancer cells. Cancer Lett. 2012;322: $113-8$.

27. Zakharia K, Miyabe K, Wang Y, Wu D, Moser CD, Borad MJ, Roberts LR. Preclinical in vitro and in vivo evidence of an antitumor effect of CX-4945, a casein kinase II inhibitor, in Cholangiocarcinoma. Transl Oncol. 2018;12:14353.

28. Song C, Gowda C, Pan X, Ding Y, Tong Y, Tan BH, Wang H, Muthusami S, Ge Z, Sachdev M, Amin SG, Desai D, Gowda K, Gowda R, Robertson GP, Schjerven H, Muschen M, Payne KJ, Dovat S. Targeting casein kinase II restores Ikaros tumor suppressor activity and demonstrates therapeutic efficacy in high-risk leukemia. Blood. 2015;126:1813-22.

29. Pierre F, Chua PC, O'Brien SE, Siddiqui-Jain A, Bourbon P, Haddach M, Michaux J, Nagasawa J, Schwaebe MK, Stefan E, Vialettes A, Whitten JP, Chen TK, Darjania L, Stansfield R, Anderes K, Bliesath J, Drygin D, Ho C, Omori M, Proffitt C, Streiner N, Trent K, Rice WG, Ryckman DM. Discovery and SAR of 5-(3-Chlorophenylamino)benzo[ C ][2,6]naphthyridine-8carboxylic acid (CX-4945), the first clinical stage inhibitor of protein kinase CK2 for the treatment of Cancer. J Med Chem. 2011;54:635-54.

30. Vainchenker W, Dusa A, Constantinescu SN. JAKs in pathology: role of Janus kinases in hematopoietic malignancies and immunodeficiencies. Semin Cell Dev Biol. 2008;19:385-93.

31. Wang K, Wei G, Liu D. CD19: a biomarker for B cell development, lymphoma diagnosis and therapy. Exp Hematol Oncol. 2012;1:36

32. Haga RB, Ridley AJ. Rho GTPases: regulation and roles in cancer cell biology. Small GTPases. 2016;7:207-21.

33. Su JL, Lin MT, Hong CC, Chang CC, Shiah SG, Wu CW, Chen ST, Chau YP, Kuo ML. Resveratrol induces FasL-related apoptosis through Cdc42 activation of ASK1/JNK-dependent signaling pathway in human leukemia HL-60 cells. Carcinogenesis. 2005;26:1-10.

34. Chuang TH, Hahn KM, Lee JD, Danley DE, Bokoch GM. The small GTPase Cdc42 initiates an apoptotic signaling pathway in Jurkat T lymphocytes. Mol Biol Cell. 1997:8:1687-98.

35. Ge Z, Zhou X, Gu Y, Han Q, Li J, Chen B, Ge Q. Ikaros regulation of the BCL6 / $\mathrm{BACH} 2$ axis and its clinical relevance in acute lymphoblastic leukemia. Oncotarget. 2017;8:8022-34.

36. Zhang $\mathrm{H}$, Chen Z, Miranda RN, Medeiros LJ, McCarty N. Bifurcated BACH2 control coordinates mantle cell lymphoma survival and dispersal during hypoxia. Blood. 2017;130:763-76.

37. Guerra B, Rasmussen TDL, Schnitzler A, Jensen HH, Boldyreff BS, Miyata Y, Marcussen N, Niefind K, Issinger O-G. Protein kinase CK2 inhibition is associated with the destabilization of HIF-1a in human cancer cells. Cancer Lett. 2015;356(2 Pt B):751-61.

38. Hubert A, Paris S, Piret J-P, Ninane N, Raes M, Michiels C. Casein kinase 2 inhibition decreases hypoxia-inducible factor-1 activity under hypoxia through elevated p53 protein level. J Cell Sci. 2006;119(Pt 16):3351-62

39. Tamahara T, Ochiai K, Muto A, Kato Y, Sax N, Matsumoto M, Koseki T, Igarashi K. The mTOR-Bach2 Cascade controls cell cycle and class switch recombination during B cell differentiation. Mol Cell Biol. 2017;37.

40. Sarbassov DD, Guertin DA, Ali SM, Sabatini DM. Phosphorylation and regulation of Akt/PKB by the rictor-mTOR complex. Science. 2005;307:1098-101.

41. Chen $\mathrm{CH}$, Hsia TC, Yeh MH, Chen TW, Chen YJ, Chen JT, Wei YL, Tu CY, Huang WC. MEK inhibitors induce Akt activation and drug resistance by suppressing negative feedback ERK-mediated HER2 phosphorylation at Thr701. Mol Oncol. 2017;11:1273-87.

42. Baumgartner C, Toifl S, Farlik M, Halbritter F, Scheicher R, Fischer I, SexI V, Bock C, Baccarini M. An ERK-Dependent Feedback Mechanism Prevents Hematopoietic Stem Cell Exhaustion. Cell Stem Cell. 2018;22:879-92 e6.

43. Hall A. Rho GTPases and the control of cell behaviour. Biochem Soc Trans. 2005;33:891.

\section{Publisher's Note}

Springer Nature remains neutral with regard to jurisdictional claims in published maps and institutional affiliations.
Ready to submit your research? Choose BMC and benefit from:

- fast, convenient online submission

- thorough peer review by experienced researchers in your field

- rapid publication on acceptance

- support for research data, including large and complex data types

- gold Open Access which fosters wider collaboration and increased citations

- maximum visibility for your research: over $100 \mathrm{M}$ website views per year

At BMC, research is always in progress.

Learn more biomedcentral.com/submissions 